\title{
Toward a Core Outcome Measurement Set for Polymyalgia Rheumatica: Report from the OMERACT 2018 Special Interest Group
}

\author{
Claire E. Owen, Max Yates, Helen Twohig, Sara Muller, Lorna M. Neill, Eileen Harrison, \\ Beverley Shea, Lee S. Simon, Catherine L. Hill, and Sarah L. Mackie
}

ABSTRACT. Objective. To report the progress of the Outcome Measures in Rheumatology (OMERACT) Polymyalgia Rheumatica (PMR) Working Group in selecting candidate instruments for a core outcome measurement set.

Methods. A systematic literature review identified outcomes measured and instruments used in PMR studies, and a respondent survey and raw data analysis assessed their domain match and feasibility. Results. Candidate instruments were identified for pain [visual analog scale/numerical rating scale (VAS/NRS)], stiffness (VAS/NRS and duration), and physical function (Health Assessment Questionnaire-Disability Index/modified Health Assessment Questionnaire). Domain match and feasibility assessments were favorable; however, validation in PMR was lacking.

Conclusion. Further assessment of candidate instruments is required prior to recommending a PMR core outcome measurement set. (First Release April 1 2019; J Rheumatol 2019;46:1360-4; doi:10.3899/jrheum.181050)

Key Indexing Terms:

POLYMYALGIA RHEUMATICA

OUTCOMES

OMERACT

From the Department of Rheumatology, Austin Health; Department of Medicine, University of Melbourne, Melbourne; Rheumatology Unit, Royal Adelaide Hospital; Discipline of Medicine, The University of Adelaide, Adelaide, Australia; Department of Rheumatology, Addenbrooke's Hospital, Cambridge; Norwich Medical School, University of East Anglia, Norwich; Arthritis Research UK Primary Care Centre, Primary Care Sciences, Keele University, Keele; PMR-GCA Scotland, Dundee, Angus; PMR-GCA North East, Kibblesworth, Tyne and Wear; Leeds Institute of Rheumatic and Musculoskeletal Medicine, University of Leeds; UK National Institute for Health Research (NIHR)-Leeds Biomedical Research Centre, Leeds Teaching Hospitals National Health Service (NHS) Trust, Leeds, UK; Ottawa Hospital Research Institute; School of Epidemiology, Public Health and Preventative Medicine, University of Ottawa, Ottawa, Ontario, Canada; SDG LLC, Cambridge, Massachusetts, USA.

C.E. Owen, MBBS (Hons), Consultant Rheumatologist, Department of Rheumatology, Austin Health, and Department of Medicine, University of Melbourne; M. Yates, PhD, Clinical Research Fellow, Department of Rheumatology, Addenbrooke's Hospital, and Norwich Medical School, University of East Anglia; H. Twohig, MBChB, General Practitioner, Arthritis Research UK Primary Care Centre, Primary Care Sciences, Keele University; S. Muller, PhD, Senior Research Fellow, Arthritis Research UK Primary Care Centre, Primary Care Sciences, Keele University; L.M. Neill, BSc, OMERACT, Patient Research Partner, PMR-GCA Scotland; E. Harrison, BSc, OMERACT, Patient Research Partner, PMR-GCA North East; B. Shea, PhD, Senior Methodologist and Adjunct Professor, Ottawa Hospital Research Institute, and School of Epidemiology, Public Health and Preventative Medicine, University of Ottawa; L.S. Simon, MD, Principal, SDG LLC; C.L. Hill, MD, Clinical Professor and Consultant Rheumatologist, Rheumatology Unit, Royal Adelaide Hospital, and Discipline of Medicine, The University of Adelaide; S.L. Mackie, PhD, Associate Clinical Professor and Consultant Rheumatologist, Leeds Institute of Rheumatic and Musculoskeletal Medicine, University of Leeds, and NIHR-Leeds Biomedical Research Centre, Leeds Teaching Hospitals NHS Trust.

Address correspondence to C.E. Owen, Rheumatology Department, Austin Health, Repatriation Campus, Level 1, North Wing, 300 Waterdale Road, Heidelberg West VIC, Australia 3081.E-mail: claire.owen@austin.org.au Accepted for publication January 17, 2019.
Polymyalgia rheumatica (PMR) is an inflammatory disease characterized by subacute onset pain and stiffness in the shoulders and hips. Oral glucocorticoids represent the mainstay of treatment and while cessation of therapy is the ultimate goal, up to $50 \%$ of patients with PMR continue to require prednisolone 2-3 years after diagnosis ${ }^{1}$. It is unclear what starting dose or tapering schedule achieves the best outcome, or what benefit may be offered by putative glucocorticoid-sparing agents. Significant morbidity from glucocorticoid-induced complications is recognized and likely surpasses that seen in comparable rheumatic conditions ${ }^{2}$.

To our knowledge, there is currently no agreed core outcome measurement set for PMR clinical trials. A lack of consistency in definitions for domains or instruments used to assess patients with PMR is characteristic of the existing literature $^{3}$. A core outcome measurement set for universal use in studies of PMR would improve the quality of future research.

In 2016, the Outcome Measures in Rheumatology (OMERACT) endorsed a core domain set for PMR. The inner core of the "onion," signifying items to be measured in all PMR clinical trials, consisted of 4 domains: pain, stiffness, physical function, and systemic inflammation ${ }^{4}$. Here we report the progress of the PMR Working Group in identifying and evaluating suitable instruments mapping to these core domains. This work includes an updated systematic literature review, online respondent survey, and raw data analysis evaluating the domain match and feasibility of selected instruments in line with the first 2 signaling questions of the OMERACT Filter 2.1: Instrument Selection Algorithm $5,6,7$. The proceedings of the OMERACT 2018 PMR Special 
Interest Group (SIG) are also detailed, in particular the major points discussed, and consensus reached regarding which candidate instruments should continue through the filter.

\section{Systematic Literature Review}

To obtain all published articles reporting outcome measures mapping to the OMERACT-endorsed PMR Core Domain Set, 5 databases (MEDLINE, CINAHL, Embase, Web of Science, Cochrane Library) were searched from inception to September 30, 2017. This yielded 16,222 references, which was reduced to 90 full-text studies following removal of duplicates and screening abstracts. Forty-six studies were included in the review. Risk of bias was assessed using a modified Quality in Prognosis Studies (QUIPS) tool ${ }^{8}$. The systematic review protocol was registered with PROSPERO (CRD42017080058).

Two out of 10 randomized controlled trials and 12/23 prospective cohort studies measured outcomes in each of the 4 core domains. The most commonly assessed domain was systemic inflammation (43/46 studies), usually by erythrocyte sedimentation rate (ESR) and/or C-reactive protein (CRP). Pain was measured in 32/46 studies, most often using a visual analog scale (VAS). Stiffness was measured in 28/46 studies, typically as duration of morning stiffness. Physical function was assessed in 22/46 of studies, most frequently using the elevation of upper limb score as part of the PMR activity score ${ }^{9}$, or the Health Assessment Questionnaire (HAQ). There was no association between higher-quality clinical research trials (assessed by QUIPS tool) and the number of outcomes measured from the core domain set.

\section{Online Respondent Survey}

The patient perspective on candidate instruments for pain (VAS/numeric rating scale [NRS]), stiffness (VAS/NRS, and duration of morning stiffness), physical function [Health Assessment Questionnaire-Disability Index (HAQ-DI)/ modified Health Assessment Questionnaire (MHAQ)], and systemic inflammation (ESR/CRP) were evaluated using a Web-based survey. Google Docs links were created and included in the Newswire newsletter of the charity PMRGCAuk (Polymyalgia Rheumatica and Giant Cell Arteritis UK), which is distributed to 1800 readers, and the HealthUnlocked Web forum consisting of 6986 members, yielding between 28 and 73 responses for each of the instruments examined. Patients were asked a series of questions addressing face validity and feasibility that had been developed by consensus of the PMR Working Group. Ethical approval was received from the University of East Anglia Research Ethics Committee (2017/18 - 81) and all respondents provided written informed consent to publish the results prior to survey completion.

Participants from 3 continents (Europe, North America, Australia/New Zealand) contributed 51 responses for pain
VAS/NRS, 51 for stiffness VAS/NRS, 51 for duration of morning stiffness, 73 for HAQ-DI, 28 for MHAQ, and 62 for ESR/CRP. Disease duration ranged from new diagnosis to 17 years; current prednisolone dose was 0-50 mg daily. Most respondents agreed candidate instruments were both a suitable match for the target domain and feasible to complete: approval for pain VAS was 68.6\%/NRS 60.8\%; stiffness VAS 62.8\%/NRS 58.8\%; duration of morning stiffness 58.8\%; HAQ-DI 70\%; MHAQ 53.6\%; and ESR/CRP 54.8\%. The free text responses further contextualized the numerical scores and will be the subject of a separate publication arising from this work.

\section{Raw Data Analysis}

Two prospective observational cohort studies contributed raw data to further assess the domain match and feasibility of candidate instruments for pain, stiffness, and physical function: the Melbourne Predictors of Relapse in PMR (MPR-PMR) study, and The PMR Cohort ${ }^{10}$. Specifics pertaining to each of these studies' designs and baseline patient demographics are outlined in Table $1^{11,12}$. Ethical approval was received from the Austin Health Research Ethics Committee for the MPR-PMR study (HREC/14/ Austin/158) and the Staffordshire Research Ethics Committee for The PMR Cohort (12/WM/0021v), with written informed consent including publication of results provided by all participants in both instances prior to study enrollment.

Statistical analysis was conducted using Stata ${ }^{11,12}$. A data completion rate $>80 \%$ was ensured for each instrument, prior to generating a frequency distribution histogram. The proportion (percentage) of participants with the lowest ("floor") and highest ("ceiling") values was recorded, and the normality of each distribution was assessed (based upon the coefficient of skewness where $0=$ normal and -0.5 to 0.5 is about symmetric).

The data completion rate $(\geq 96.3 \%)$ and time taken were deemed acceptable for all of the candidate instruments examined. Pain VAS/NRS and stiffness NRS were both associated with $>15 \%$ of respondents scoring the highest possible value $(\mathrm{VAS} / \mathrm{NRS}=10)$ at baseline, and the lowest value $(\mathrm{VAS} / \mathrm{NRS}=0)$ at multiple timepoints during treatment and followup. Both versions of the HAQ, but especially the MHAQ, were similarly characterized by floor effects (lowest value $=0$ ) throughout but did not show the same ceiling problems (highest value $=3$ ). The observed floor and ceiling patterns appeared consistent with the expected clinical course for patients with newly diagnosed PMR.

At baseline in both studies, pain levels were at the higher end of the scale (VAS/NRS), then appropriately trended to lower values following treatment (Figure 1). While the MPR-PMR study measured duration of morning stiffness, as compared with stiffness NRS in The PMR Cohort, the pattern of distribution was similar for both instruments and mirrored

Personal non-commercial use only. The Journal of Rheumatology Copyright (c) 2019. All rights reserved. 
Table 1. Study design and baseline demographic data for the MPR-PMR study and The PMR Cohort.

\begin{tabular}{|c|c|c|}
\hline Criterion & MPR-PMR Study & The PMR Cohort \\
\hline Study type & Prospective, observational & Prospective, observational \\
\hline Setting & Tertiary referral center & Primary care \\
\hline Mode of survey distribution & Clinician administered & Mail \\
\hline Sample size, $\mathrm{n}$ & 37 & 652 \\
\hline Mean age, $y r s \pm S D$ & $62.9 \pm 24.6$ & $72.4 \pm 9.3$ \\
\hline Female & $46.0 \%$ & $62.2 \%$ \\
\hline White & $97.3 \%$ & $97.0 \%$ \\
\hline Diagnostic criteria & 2012 EULAR/ACR ${ }^{11}$ & Clinician diagnosis \\
\hline Treatment & BSR guideline ${ }^{12}$ & Clinician discretion \\
\hline Visit schedule & Weeks $0,4,8,16,24,32$, and 46 & Mos $0,1,4,8,12,18$, and 24 \\
\hline Followup duration & 46 weeks & $24 \mathrm{mos}$ \\
\hline Instrument used to measure pain & VAS & NRS \\
\hline Pain VAS/NRS stem and anchors & $\begin{array}{l}\text { Please indicate on the scale below the } \\
\text { severity of your current muscle/joint pain } \\
\text { (where 0 is "no pain" and } 10 \text { is "pain as } \\
\text { bad as it could be"). }\end{array}$ & $\begin{array}{c}\text { How would you rate the } \\
\text { overall level of pain from } \\
\text { your PMR on a } 0-10 \text { scale, } \\
\text { where } 0 \text { is "no pain" and } \\
10 \text { is "pain as bad as it } \\
\text { could be"? }\end{array}$ \\
\hline Instrument used to measure stiffness & Duration of morning stiffness, min & NRS \\
\hline Instrument used to measure physical $\mathrm{f}$ & action $\quad$ HAQ-DI & MHAQ \\
\hline
\end{tabular}

PMR: polymyalgia rheumatica; MPR-PMR: Melbourne Predictors of Relapse-Polymyalgia Rheumatica; MPRPMR: Melbourne Predictors of Relapse-PMR; EULAR/ACR: European ; EULAR/ACR: European League Against Rheumatism/American College of Rheumatology; BSR: British Society of Rheumatology; VAS: visual analog scale; NRS: numerical rating scale; HAQ-DI: Health Assessment Questionnaire-Disability Index; MHAQ: modified Health Assessment Questionnaire.
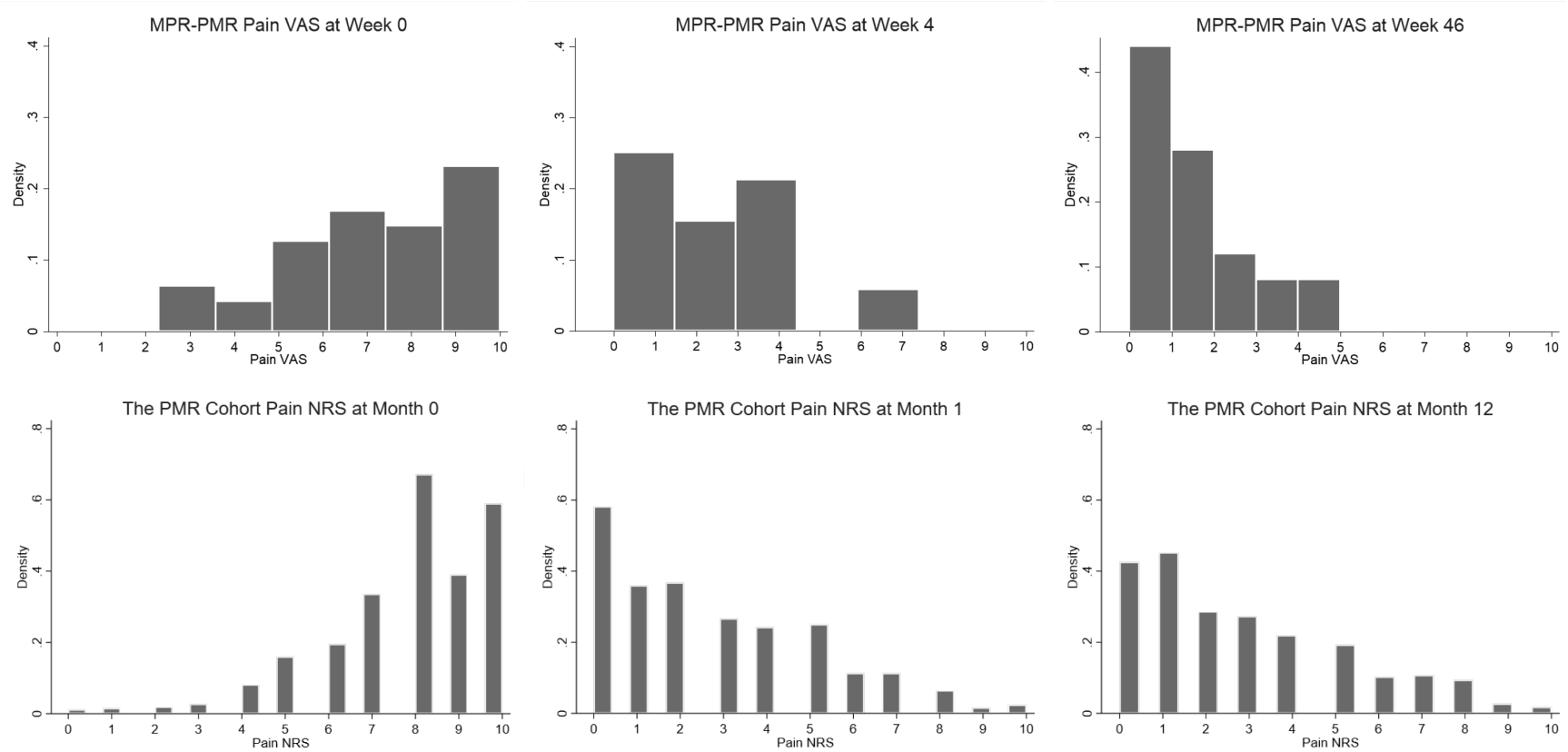

Figure 1. Frequency distribution histograms for pain VAS in the MPR-PMR study at weeks 0,4 , and 46 compared with pain NRS in The PMR Cohort at months 0,1 , and 12. On the Y axis, density refers to the average frequency of scores within the allocated interval. VAS: visual analog scale; MPR-PMR: Melbourne Predictors of Relapse-Polymyalgia Rheumatica; NRS: numerical rating scale.

that seen for pain VAS/NRS. MHAQ results at baseline in The PMR Cohort were typically lower than those recorded using HAQ-DI, which might be explained by the shorter format of this instrument. During followup, no major differences were noted in the performance of the HAQ-DI compared with MHAQ (Figure 2). 

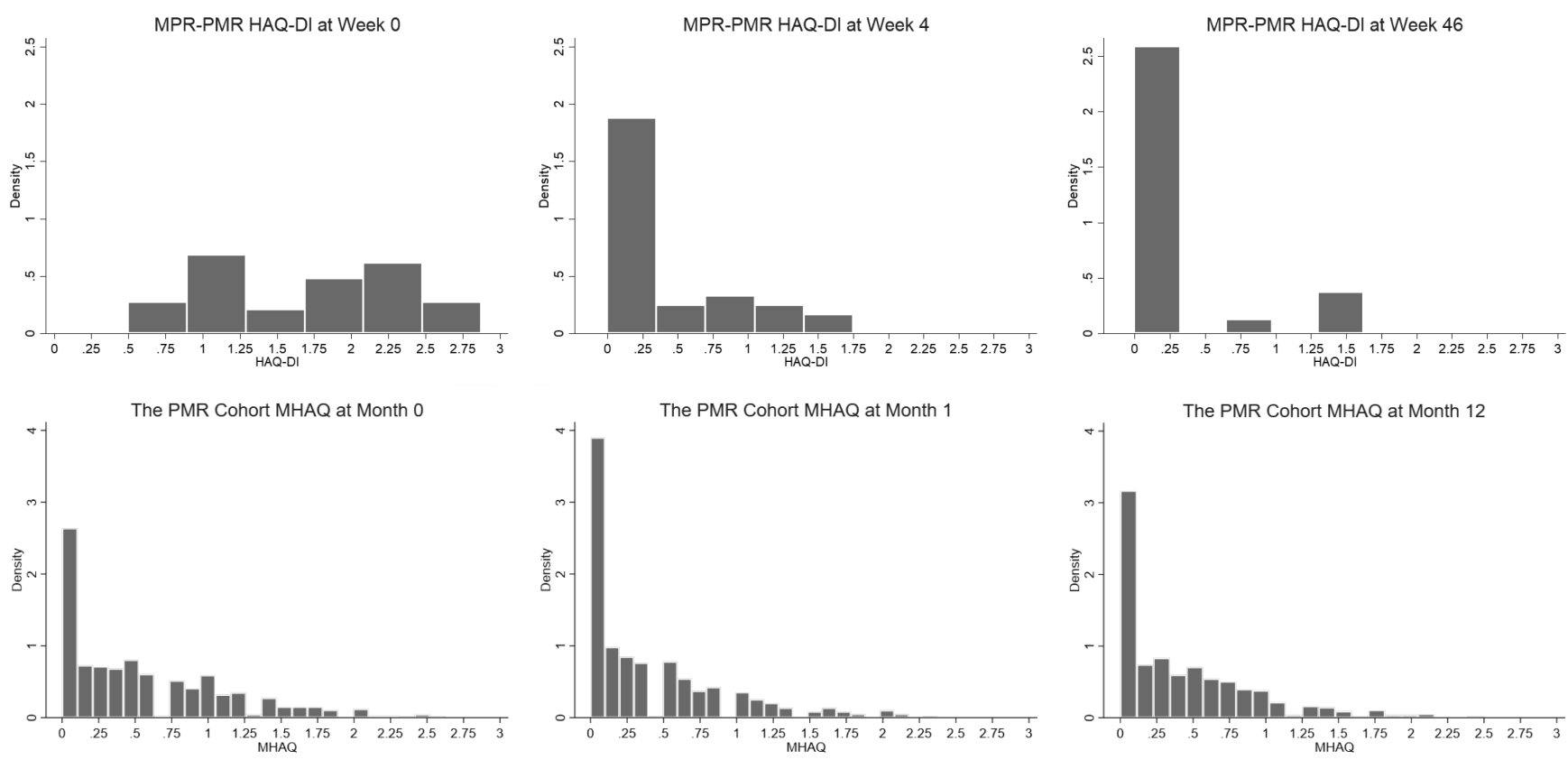

Figure 2. Frequency distribution histogram for HAQ-DI in the MPR-PMR study at weeks 0, 4, and 46 compared with MHAQ in The PMR Cohort at months 0,1 , and 12 . On the Y axis, density refers to the average frequency of scores within the allocated interval. HAQ-DI: Health Assessment Questionnaire-Disability Index; MPR-PMR: Melbourne Predictors of Relapse-Polymyalgia Rheumatica; MHAQ: modified Health Assessment Questionnaire.

\section{Summary of the OMERACT 2018 PMR SIG}

Participants including clinicians, researchers, and patient partners discussed the results of the 3 work streams in detail at the OMERACT 2018 PMR SIG. The purpose of the SIG was to establish whether instruments mapping to the 4 core domains had satisfied tests for domain match and feasibility, and if they should continue through the OMERACT 2.1 Filter $5,6,7$.

The major points of discussion were the interchangeability of VAS and NRS for the measurement of pain, the most suitable instrument to measure stiffness (VAS/NRS vs duration of morning stiffness), and the appropriateness of HAQ as a patient-reported outcome measure (PROM) across different age groups and in the modern day. Although the raw data analysis revealed no major differences in the performance of pain VAS and NRS in the 2 PMR populations studied, no head-to-head comparison was available, and this may require additional study. VAS/NRS and duration of morning stiffness were both acknowledged to possess limitations in their respective abilities to measure the patient experience of stiffness (particularly when eliciting responses from non-English speaking patients); this issue is common to many rheumatic diseases and no better alternative for measuring stiffness in PMR is currently described. While some HAQ questions may be less relevant to older persons (e.g., "Do chores such as vacuuming or yard work?") or contextually out of date (e.g., "run errands?"), it is otherwise a well-validated instrument that in other diseases has been shown to be responsive to change over time and capable of discriminating between groups of interest. The development of an entirely new instrument for the domain of physical function was therefore deemed unnecessary. However, it is recognized that the overall life impact of PMR reaches beyond the 4 core domains and there remains an unmet need for a disease-specific PROM for PMR.

\section{Future Research Agenda}

At the end of the SIG, consensus was reached among the participants that candidate instruments for pain (VAS/NRS), stiffness (VAS/NRS and duration of morning stiffness), and physical function (HAQ-DI/MHAQ) were either green ("good to go") or amber ("more work needed or a concern, but go") for domain match and feasibility. The PMR Working Group will focus upon appraising the existing evidence for each instrument's measurement properties before addressing any identified gaps by undertaking focused analysis of relevant datasets. Our objective is to generate a PMR Core Outcome Measurement Set for future endorsement by OMERACT.

\section{REFERENCES}

1. Kremers HM, Reinalda MS, Crowson CS, Zinsmeister AR, Hunder GG, Gabriel SE. Relapse in a population based cohort of patients with polymyalgia rheumatica. J Rheumatol 2005;32:65-73.

2. Hoes JN, Jacobs JW, Verstappen SM, Bijlsma JW, Van der Heijden GJ. Adverse events of low- to medium-dose oral glucocorticoids in inflammatory diseases: a meta-analysis. Ann Rheum Dis 2009;68:1833-8.

Personal non-commercial use only. The Journal of Rheumatology Copyright $\subset$ 2019. All rights reserved. 
3. Duarte C, Ferreira RJ, Mackie SL, Kirwan JR, Pereira da Silva JA; OMERACT Polymyalgia Rheumatica Special Interest Group.

Outcome measures in polymyalgia rheumatica. A systematic review. J Rheumatol 2015;42:2503-11

4. Mackie SL, Twohig H, Neill LM, Harrison E, Shea B, Black RJ, et al; OMERACT PMR Working Group. The OMERACT core domain set for outcome measures for clinical trials in polymyalgia rheumatica. J Rheumatol 2017;44:1515-21.

5. Boers M, Kirwan JR, Wells G, Beaton D, Gossec L, d'Agostino MA, et al. Developing core outcome measurement sets for clinical trials: OMERACT filter 2.0. J Clin Epidemiol 2014;67:745-53.

6. Boers M, Kirwan JR, Tugwell P, Beaton D, Bingham CO III, Conaghan PG, et al. The OMERACT Handbook. [Internet. Accessed May 17, 2017.] Available from: https://omeract.org/resources
7. Beaton D, Maxwell LJ, Shea B, Wells GA, Boers M, Grosskleg S, et al. Instrument selection using the OMERACT Filter 2.1: The OMERACT methodology. J Rheumatol 2019 (accepted).

8. Hayden JA, van der Windt DA, Cartwright JL, Côté P, Bombardier C. Assessing bias in studies of prognostic factors. Ann Intern Med 2013;158:280-6.

9. Leeb B, Bird H. A disease activity score for polymyalgia rheumatica. Ann Rheum Dis 2004;63:1279-83.

10. Muller S, Hider S, Helliwell T, Bailey J, Barraclough K, Cope L, et al. The epidemiology of polymyalgia rheumatica in primary care: a research protocol. BMC Musculoskelet Disord 2012;13:102.

11. StataCorp. Stata statistical software: Release 13. College Station, TX: StataCorp LP; 2013.

12. StataCorp. Stata statistical software: Release 15. College Station, TX: StataCorp LLC; 2017. 